\title{
Evaluation of radiative transfer schemes for mesoscale model data assimilation: a case study
}

\author{
A. Memmo ${ }^{1}$, C. Faccani ${ }^{1}$, R. Ferretti ${ }^{1,2}$, S. Di Michele ${ }^{3}$, and F. S. Marzano ${ }^{1,4}$ \\ ${ }^{1}$ Centro di Eccellenza CETEMPS, Università dell'Aquila, L'Aquila, Italy \\ ${ }^{2}$ Dipartimento di Fisica, Università dell' Aquila, L'Aquila, Italy \\ ${ }^{3}$ Research Department, Satellite Section, European Centre for Medium-Range Weather Forecasts (ECMWF), Reading, UK \\ ${ }^{4}$ Dipartimento di Ingegneria Elettronica Università di Roma "La Sapienza", Rome, Italy
}

Received: 11 November 2005 - Revised: 23 December 2005 - Accepted: 13 January 2006 - Published: 28 February 2006

\begin{abstract}
The assimilation of Special Sensor Microwave Imager (SSM/I) data into the Mesoscale Model 5 (MM5) allows for improving the weather forecast. However the results suggested an update the Radiative Transfer Equation (RTE) within the three-dimensional variational (3DVAR) algorithm which is tailored for non rainy conditions only. To this purpose, a new RTE algorithm is tested, in order to account for radiometric response in rainy regions. The new brightness temperatures $\left(T_{B}\right)$ are estimated by using hydrometeor profiles from the MM5 mesoscale model, running with two different microphysical parameterizations. The goodness of the results is assessed by comparing the new $T_{B}$ with those of the original RTE algorithm in the 3DVAR code and the SSM/I observed data. The results confirm a better reliability of the new RTE compared to the old one.
\end{abstract}

\section{Introduction}

The variational data assimilation is one of the major issues for the scientific meteorological community (Courtier et al., 1998; Gustafsson et al., 2001; Barker et al., 2004) being a powerful technique to ingest a large variety of measurements into numerical weather forecast models. In this context, satellite data can provide a large amount of information, especially over those areas (sea surface) where conventional observations, such as radiosonde or synop, are not available or scarce. Satellite data are also fundamental to improve the initialization of the numerical weather prediction models.

Particularly, the Special Sensor Microwave Imager (SSM/I) can provide information on the atmospheric moist variables, such as precipitable water, cloud liquid water and rain rate over the sea surface retrieved from measured brightness temperatures $\left(T_{B}\right)$. The latter may also be assimilated

Correspondence to: A. Memmo

(adelaide.memmo@aquila.infn.it) directly, but this task requires the set up of an accurate Radiative Transfer Equation (RTE) algorithm which, in away, maps the three-dimensional (3-D) gaseous concentration and atmospheric particles distribution into a radiance field (Bauer et al., 2002). This mapping is easily obtained in clear sky or non rainy conditions (Faccani et al., $2005^{1}$ ), but it becomes complex in rainfall conditions (Amerault and Zou, 2003). The computation of the atmospheric microwave radiance in cloudy and rainy conditions is a critical task for radiative transfer models, as it has to include multiple scattering effects (Marzano et al., 1999a, 1999b; Di Michele et al., 2005). To accurately compute $T_{B}$ in previous conditions, it is necessary to use a hydrometeor profiles such as liquid water droplets, raindrops, ice crystals, snow and graupel particles. Unfortunately, this information is not available through conventional observations, so that a simulated environment is often set up by resorting to a numerical weather forecast model (Bauer, 2000; Kummerow et al., 2001; Tassa et al., 2003). The numerical model has to provide a detailed microphysical description with a fine spatial resolution both in time and space.

In this work an RTE scheme, based on the pseudo-3-D Eddington algorithm (RTE-EDD), is tested for variational data assimilation purposes into mesoscale forecast models. The RTE algorithm can handle rainy areas and uses a hydrometeor profile extracted from the Mesoscale Model 5 (MM5 from NCAR/PSU) non-hydrostatic model. Two different microphysical parameterizations of hydrometeor distribution are selected within MM5. The $T_{B}$ simulations are compared with the SSM/I observations and with those computed using the standard RTE algorithm coded for the three-dimensional Variational (3DVAR) data assimilation scheme.

\footnotetext{
${ }^{1}$ Faccani, C., Ferretti, R., Cimini, D., and Marzano, F. S.: Three-dimensional variational assimilation of Special Sensor Microwave/Imager data on mesoscale weather prediction model: a case study, in preparation, 2005.
} 


\section{Simulation of microwave radiometric response}

A general theoretical framework to model the up-welling brightness temperature is given by the radiative transfer theory (Ulaby et al., 1981). For a plane-parallel geometry, the unpolarized azimuthally-symmetric up-welling brightness temperature can be formally expressed by means from radiative transfer equation (RTE), that is:

$$
\begin{aligned}
& \frac{d T_{B}(\tau, \Omega)}{d \tau}=-T_{B}(\tau, \Omega)+\frac{w}{4 \pi} \int_{4 \pi} p\left(\Omega, \Omega^{\prime}\right) T_{B}(\tau, \Omega) d \Omega^{\prime} \\
& +(1-w) T(\tau)
\end{aligned}
$$

where $\tau$ is the optical thickness $\Omega$ is the solid angle, $p\left(\Omega, \Omega^{\prime}\right)$ is the phase function and $w$ is the volumetric albedo and where $J\left(\tau_{\nu} \mu\right)$ is the pseudo source function given by:

$J\left(\tau_{\nu}, \mu\right)=\frac{w}{4 \pi} \int_{4 \pi} p\left(\Omega, \Omega^{\prime}\right) T_{b}\left(\tau_{\nu}, \Omega^{\prime}\right) d \Omega^{\prime}+[1-w] T\left(\tau_{\nu}\right)$

with $w$ the volumetric albedo, $p\left(\Omega, \Omega^{\prime}\right)$ the volumetric azimuthally-averaged scattering phase function, and $T$ the physical temperature. The first term of $J$ is sometimes referred to as a multiple scattering source, while the second term represents thermal emission. The atmosphere is assumed to consist of adjacent homogeneous layers in which volumetric albedo $w$, extinction coefficient $k_{e}$ and phase function $p$ are taken to be constant within layers. The atmospheric gas and hydrometeor profiles can be derived from mesoscale cloud models at high spatial resolution such as the MM5 model.

\subsection{Mesoscale cloud numerical model}

The MM5 mesoscale model from PSU/NCAR (Grell et al., 1994; Dudhia, 1993) is used for the experiments; the model is running with 29 vertical levels, $27 \mathrm{~km}$ of grid step in region lat $=[35,50]-$ lon $=[-3,23]$. Two different experiments are performed using two microphysical parameterizations: Reisner 1 (R1) (Reisner et al., 1998) and Goddard (GSFC) microphysics (Tao and Simpson, 1993).

The R1 scheme accounts for cloud water $\left(q_{w}\right)$, cloud ice $\left(q_{i}\right)$, rain $\left(q_{r}\right)$ and snow $\left(q_{s}\right)$ to be produced simultaneously, but it does not account for the production of graupel. Instead, the GSFC scheme includes one further prognostic equation for graupel $\left(q_{g}\right)$. For both schemes the dimensional distribution of the hydrometeors is assumed to follow the MarshallPalmer distribution (Marshall and Palmer, 1948)

$N_{h} d x=N_{h}^{0} e^{-\lambda x} d x$

where $x$ is the particle size, $h$ is a generic hydrometeor, $\lambda$ is the slope parameter and $N^{0}$ is the intercept parameter. $\lambda$ is inversely proportional to the mixing ratio of the any hydrometeor $\left(q_{h} \equiv q_{w}, q_{i}, q_{r}, q_{s}\right.$, or $\left.q_{g}\right)$ :

$$
\lambda=\left(\frac{\pi \rho_{h} N_{h}^{0}}{\rho q_{h}}\right)^{1 / 4}
$$

where $\rho$ is the density of air and $\rho_{h}$ is the density of any hydrometeor. That is: the greater the mixing ratio, the wider is the size distribution. The intercept parameters $N_{h}^{0}$ are fixed for both the R1 and GSFC schemes. These two schemes also differ for a few constants which describe the concentration number of hydrometeors as well as their density. All the model simulations are initialized using ECMWF data analyses.

\subsection{RTE model in 3DVAR scheme}

The RTE algorithm in the 3-Dimensional Variational Data Assimilation (3DVAR) for MM5 is based on the Petty's algorithm (Petty, 1992, 1997). This algorithm, named RTE3DVAR, solves the problem for a non scattering atmosphere as viewed from satellite, taking into account both downwelling and upwelling radiance as well as sea surface reflection with foam effects. Moreover, it takes into account the gaseous absorption of the atmosphere using a frequency dependent parameterization derived from the comparison of the line-by-line Liebe (1985) model.

Cloud emission is also empirically parameterized in a nonlinear way to liquid water content cloud layer of known height, derived from MM5 field. Because the algorithm does not include the scattering processes the raining data are filtered out. Furthermore, because the land surface emissivity contaminates SSM/I measurements, only data over the sea surface are considered.

\subsection{RTE multiple-scattering model}

The second-order Eddington approximation is valid for linearly-polarized radiation, azimuthally isotropic, nondepolarizing and stratified medium, with linear temperature gradient and proper boundary conditions. The RTE-EDD is decomposed into (Marzano et al., 2002; Smith et al., 2002):

$$
\begin{aligned}
& \frac{d T_{B o}\left(\tau_{v}\right)}{d \tau}=-(1-w g) T_{B 1}\left(\tau_{v}\right) \\
& \frac{d T_{B 1}\left(\tau_{v}\right)}{d \tau}=-3(1-w g)\left[T_{B 0}\left(\tau_{v}\right)-\left(b_{o}+b_{1} \tau_{v}\right)\right]
\end{aligned}
$$

where $T_{B 0}$ and $T_{B 1}$ are the zero- and first-order Legendre expansions of $T_{B}$ and temperature is linearly expanded in $\mathrm{t}$ through $b_{0}$ and $b_{1}$.

The RTE-EDD model is based on the Eddington scheme for the $T_{B}$ approximation on slanted cloud geometry. It uses a cloud model procedure derived from microwave spaceborne radiometry (Marzano et al., 1999a, b; Di Michele et al., 2005) and cloud-resolving microphysical 3-D outputs. For the radiative signature generation the RTE model uses spherical-shape parameterized Particle Size Distribution (PSD) with mixed-phase melting layer, Lambertian and Fresnel surface mode, microwave bands range $5-180 \mathrm{GHz}$ and elevation angle range $0-90^{\circ}$.

\section{Comparison with measurements}

A comparison is performed between SSM/I data and both RTE-3DVAR and RTE-EDD, using the two MM5 

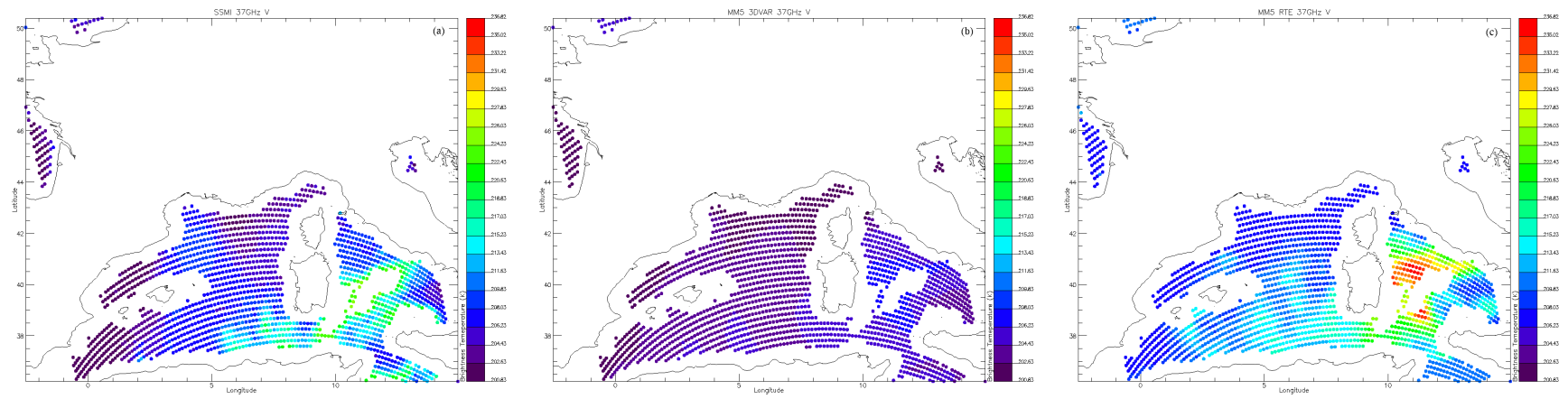

Fig. 1. Data over the sea filtered by 3D-VAR. (a) SSM/I satellite data at $37 \mathrm{GHz}$ V channel at 19:00 UTC ; (b) RTE-3DVAR simulated emission with GSFC parameterization at $37 \mathrm{GHz}$ V channel at 19:00 UTC; (c) RTE-EDD simulated emission with GSFC parameterization at $37 \mathrm{GHz} \mathrm{V}$ channel at 19:00 UTC.
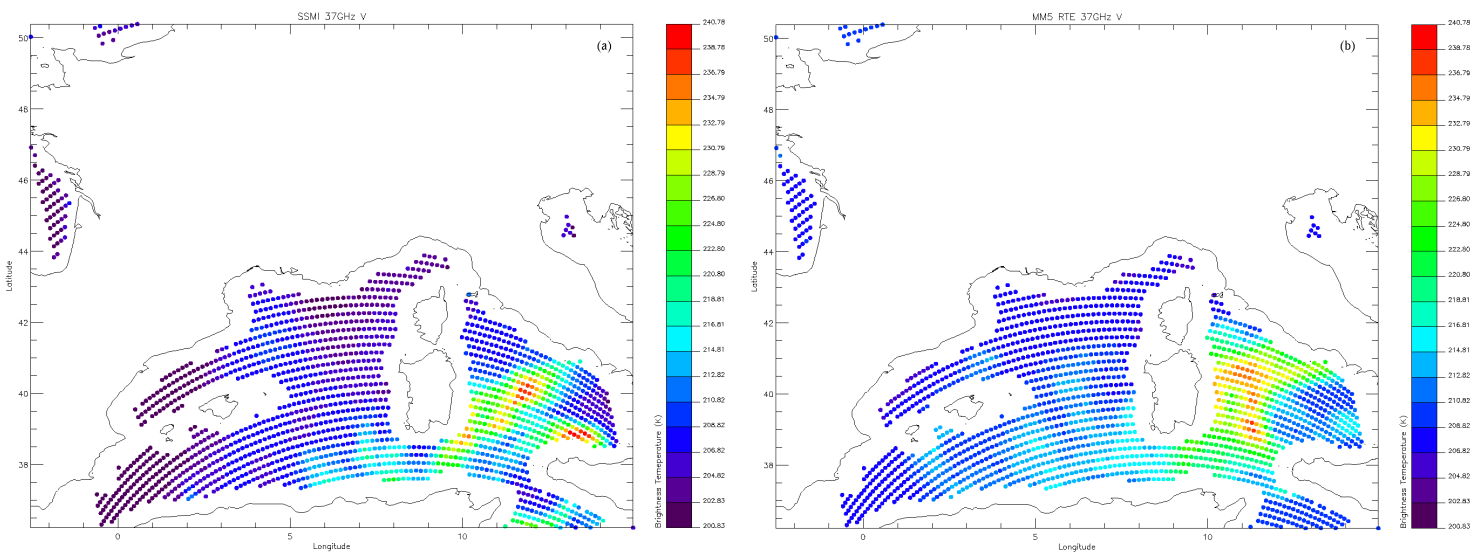

Fig. 2. All data over the sea. (a) SSM/I satellite data at $37 \mathrm{GHz}$ V channel at 19:00 UTC; (b) RTE-EDD simulated emission with GSFC parameterization at $37 \mathrm{GHz} \mathrm{V}$ channel at 19:00 UTC.

microphysical parameterizations R1 and GSFC. To the aim of understanding the impact of the assumption of non rainy conditions for the RTE-3DVAR, two different set of data are analyzed for the same SSM/I swaths: the first one is made up of excluding the rainy area, for both RTE schemes; the second one is composed of including the rainy area, but using only the Eddington RTE model.

\subsection{Satellite data}

The SSM/I data available during 24 January 2003 are used for testing both RTE and the 3DVAR algorithm. The SSM/I microwave radiometer operates on the DMSP satellites. It has four frequencies at $19.3,22.2,37.0$, and $85.5 \mathrm{GHz}$ with dual polarization, except the $22.2 \mathrm{GHz}$ frequency, which works on vertical polarization only. It has a conical scan and a swath of $1400 \mathrm{~km}$. On each scan, it takes 128 uniformly spaced samples at the $85 \mathrm{GHz}$ frequency with a spatial resolution of $12.5 \mathrm{~km}$. The others frequencies are sampled with a double temporal and spatial resolution, i.e. $8.4 \mathrm{~ms}$ and $25 \mathrm{~km}$ (Hollinger et al., 1990).

The MM5-3DVAR routine filter the measured data for rainy area so that only clear-sky and cloudy regions are se- lected. Figure 1a shows an example of SSM/I data filtered by 3DVAR routine as compared to $T_{B}$ simulations derived from RTE-3DVAR (Fig. 1b) and from RTE-EDD (Fig. 1c). If all the SSM/I data are retained (see Fig. 2a, yellow-red area), it clearly appears a region of liquid water also for the RTE-EDD (see Fig. 2b yellow-red area).

To better understand and quantify the results, histograms are built for each SSM/I channel, where the number of points that fall in a given $T_{B}$ range are counted for all time-steps. This approach is used for a preliminary evaluation and as an objective method to compare the different RTE schemes, obviously more events have to be accounted for gathering a significant statistical evaluation.

\subsection{Sensitivity to the model parameterizations}

In general, the main difference between Reisener-1 and Goddard parameterizations yield a different spatial distribution of water type particles, but this does not affect significantly the histograms of each $T_{B}$ channel (not shown). Indeed, a small difference is found at $85 \mathrm{GHz}$ channel only for the non rainy areas, suggesting that GFSC may provide a slightly better agreement with SSM/I data. This is probably due to 

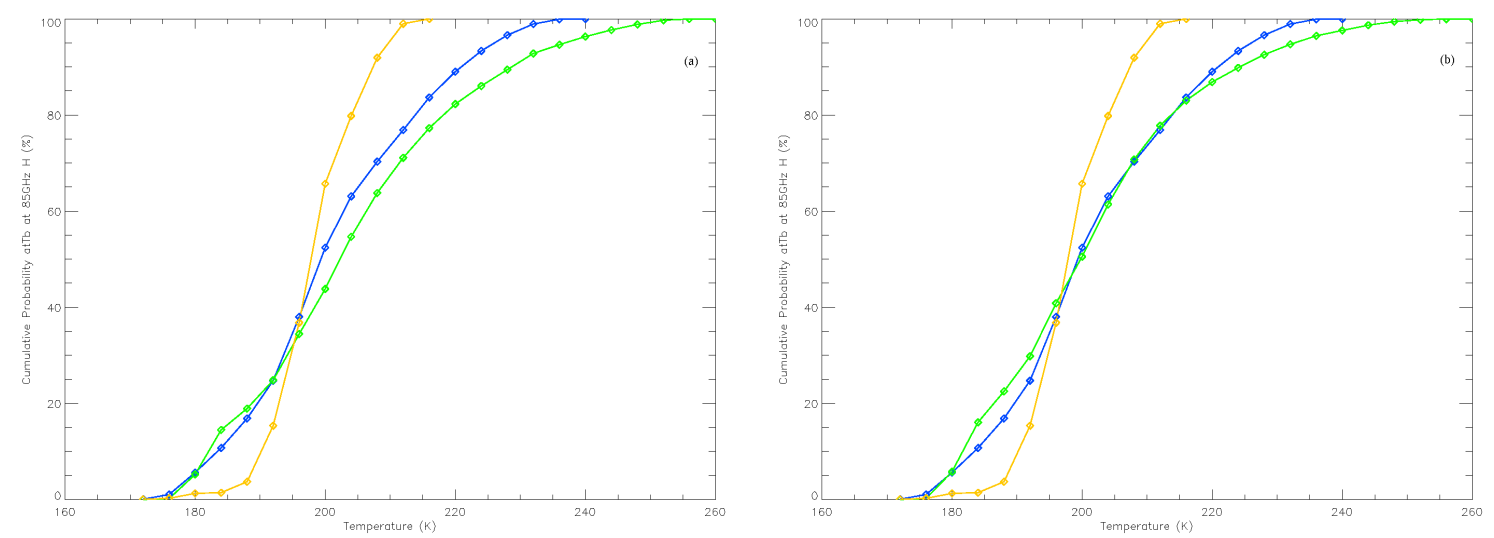

Fig. 3. Cumulative probability at $85 \mathrm{GHz} \mathrm{H}$ for (a) Reisner 1; (b) Goddard parameterizations. The color code: Yellow represents RTE3DVAR; green represents RTE-EDD and blue SSM/I observations.

the physical description adopted for graupel particles (see Fig. 3). The analysis of all $T_{B}$ channel is discussed in the following paragraphs, but only the 37 and $85 \mathrm{GHz}$ will be shown.

\subsubsection{Analysis of $19 \mathrm{GHz}$ channels}

The major contribution to the atmospheric emission at $19 \mathrm{GHz}$ is produced by the water vapour molecules. The RTE-EDD algorithm both for vertically (V) and horizontally (H) polarized channels shows a slightly larger range than RTE-3DVAR. Moreover, larger similarities are found between RTE-EDD and SSM/I data at 19V than between SSM/I observations and RTE-3DVAR (not shown). However, RTEEDD shows a clear under-estimation of $T_{B}$ at $19 \mathrm{H}$, this feature in not found for the data derived by the RTE-3DVAR algorithm (not shown).

\subsubsection{Analysis of $22 \mathrm{GHz}$ channel}

Similarly, at the $22 \mathrm{~V}$ the shape of percentage values is better reproduced by RTE-EDD algorithm than the RTE3DVAR. Moreover, both for Eddington approximation and RTE-3DVAR there is a small overestimation of the $T_{B}$ values and the former clearly does not reproduce the lower $T_{B}$ values (not shown).

\subsubsection{Analysis of $37 \mathrm{GHz}$ channel}

The $37 \mathrm{GHz}$ channels are very sensible to the liquid water particles. At the vertical channel, the RTE-EDD algorithm seems to follow the distribution of SSM/I measurements, except for lower $T_{B}$ values (Fig. 4a). This clearly shows a shifting of $T_{B}$ toward high emissions by RTE-EDD, producing an overestimation with respect to the observed one. On the other hand, the RTE-3DVAR algorithm concentrates more than $80 \%$ of its $T_{b}$ estimations in one binned interval only, centred between $202 \mathrm{~K}$ and $206 \mathrm{~K}$. This suggests a poor efficiency in reproducing the cloud emission. At $37 \mathrm{H}$, the RTE3DVAR algorithm shows a slightly more variability in $T_{B}$ values than at $\mathrm{V}$ channel, but it still produces a small range of variability centred around $142 \mathrm{~K}$ (Fig. 4b). The Eddington RTE algorithm shows an overestimation of the number of points at low temperature and an underestimation of those at high temperature (Fig. 4b). If rainy regions are taken into account, the agreement between SSM/I observations and the RTE-EDD simulations reduces especially at $\mathrm{H}$ channels in respect with the previous case. A spreader distribution is found for the RTE-EDD showing a good agreement with the observed $T_{B}$, but at lower temperature, the RTE-EDD overestimates the number of points. The results on rainy areas suggest a difficulty of the RTE-EDD model to correctly reproduce the temperature in non rainy regions (Figs. 4c, d).

\subsubsection{Analysis of $85 \mathrm{GHz}$ channel}

The major contribute to the atmospheric emission at $85 \mathrm{GHz}$ are due to solid crystals and graupel particles. At this frequency the differences between the $\mathrm{V}$ and $\mathrm{H}$ channels are remarkable for the two RTEs. At V channel, the RTE-EDD shows an overestimation of points with high $T_{B}$ values with respect to SSM/I data (top end middle in Fig. 5a); on the contrary, RTE-3DVAR shows (bottom in Fig. 5a) a distribution of brightness temperature values slightly sharper than the observed one, whose values are comparable to the low temperature of the observed SSM/I. For H channel, RTEEDD shows a good agreement with SSM/I data (top and middle in Fig. 5b), whereas RTE-3DVAR does not seem to be able to correctly reproduce the distribution of the $T_{B}$ (bottom in Fig. 5b). Indeed, RTE-3DVAR concentrated on few bins its distribution of the brightness temperature No remarkable change are found if rainy are considered.

\section{Conclusions and future developments}

The previous results infer that $T_{B}$ produced by the RTEEDD scheme assume a wide range of variability in respect with the ones produced by RTE-3DVAR, showing a better agreement (i.e. the histograms) with the SSM/I observations 

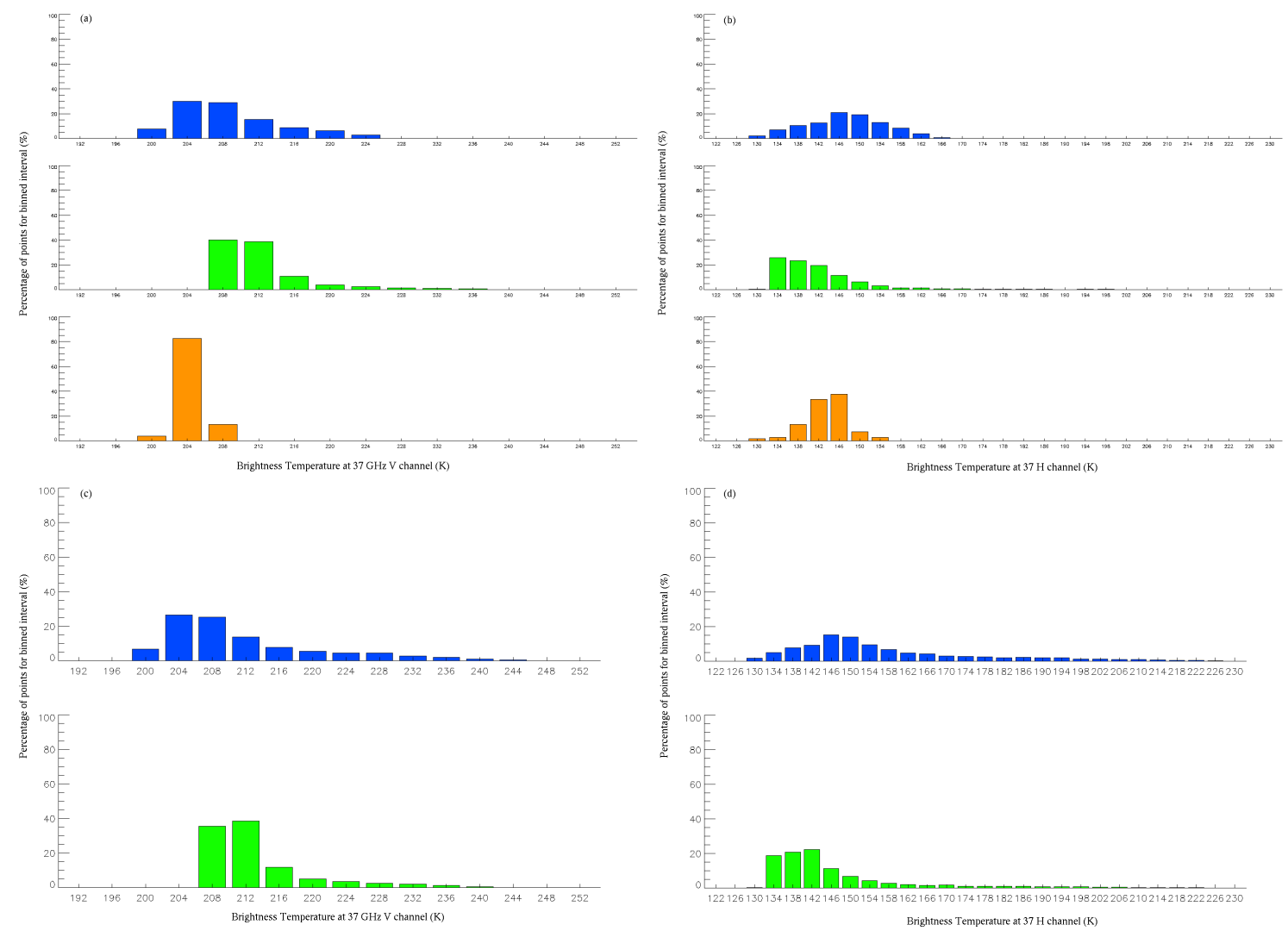

Fig. 4. Percentage of points falling in the $T_{B}$ binned interval for $37 \mathrm{GHz}$ channels for GSFC parameterization. In panel (a) is showed the $37 \mathrm{~V}$ channel for non rainy zone; in panel (b) is showed $37 \mathrm{H}$ channel for non rainy zone; in panel (c) is showed the $37 \mathrm{~V}$ channel for rainy and non rainy zone; in panel (d) is showed the $37 \mathrm{H}$ channel for rainy and non rainy zone. The color code: orange represents RTE-3DVAR; green represents RTE-EDD and blue SSM/I observations.
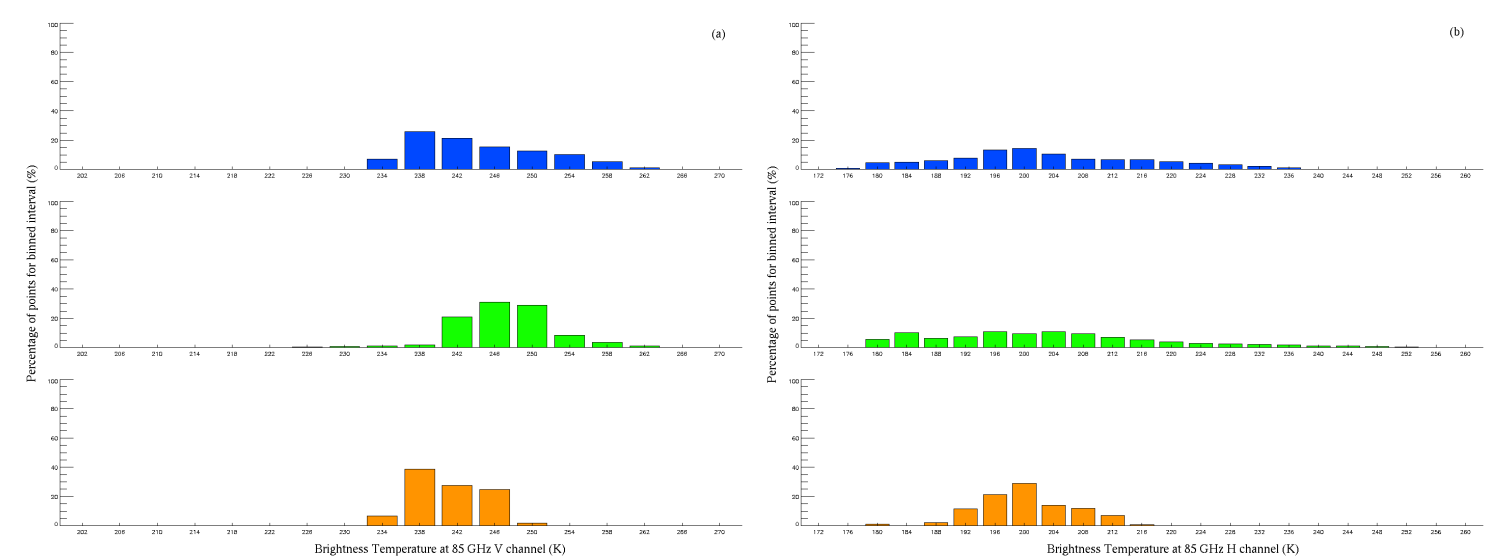

Fig. 5. Percentage of points falling in the $T_{B}$ binned interval for $85 \mathrm{GHz}$ channels for GSFC parameterization. In panel (a) is showed the $85 \mathrm{~V}$ channel for non rainy zone and in panel (b) is showed the $85 \mathrm{H}$ channel for non rainy zone. The color code: orange represents RTE-3DVAR; green represents RTE-EDD and blue SSM/I observations.

than RTE-3DVAR. Particularly, the agreement between RTEEDD and SSM/I measurements is very good for $85 \mathrm{H}$ channel; this is produced by the RTE-EDD algorithm which better describes the microphysical processes than the RTE-3DVAR one. Moreover, the RTE-3DVAR shows a generally sharper distribution of $T_{B}$ than the SSM/I observations. Finally, a tendency to underestimate the $T_{B}$ values of the horizontal polarized channels and an overestimation of the vertical ones is found for RTE-EDD. 
It has to be noticed that these results suffers of several limitations: 1) lack of observed microphysical particles ( $q_{w}$, $q_{i}, q_{r}, q_{s}$, or $\left.q_{g}\right)$; 2) MM5 mesoscale forecast-model errors which do not allow to correctly reproduce the timing and the spatial distribution of the microphysical particles, which are key parameters for a good estimation of $T_{B}$ (Amerault and Zou, 2003); 3) 3DVAR intrinsic errors; 4) RTE model approximations. All these errors are difficult to separate, this does not allow for a complete understanding of the previous findings.

Nevertheless, it has to remind that this work has been done on a one-day case study only. Therefore, even if a deeper analysis is necessary, the results are encouraging.

Acknowledgements. This work has been partially funded by INTERREG III-B CADSES project RiskAWARE and by the Region Abruzzo, Directorate of Civil Protection and Public Works.

Edited by: V. Kotroni and K. Lagouvardos

Reviewed by: anonymous referee

\section{References}

Amerault, C. and Zou, X.: Preliminary steps in assimilating SSM/I brightness temperature in a hurricane prediction scheme, J. Atmos. Oce. Tech., 20, 1154-1169, 2003.

Barker, D. M., Huang, W., Guo, Y. R., Bourgeois, A., and Xiao, Q.: Three-Dimensional Variational Data Assimilation System for MM5: Implementation and initial results, Mon. Wea. Rev., 132, 897-914, 2004.

Bauer, P., Kahib, A.,Pokrovsy, A., Meneghini, R., Kummerow, C., Marzano, F. S., and Poires Baptista, J. P. V.: Combined cloudmicrowave radiative transfer modelling of stratiform rainfall, J. Atmos. Sci., 57, 1082-1104, 2000.

Bauer, P., Mahfouf, J. F., Olson, W. S., Marzano, F. S., Di Michele, S., Tassa, A., and Mugnai, A.: Erron analysis in rainfall retrieval for variational data assimilation, Q. J. R. Meteorol. Soc., 128, 2129-2144, 2002.

Courtier, P., Anderson, E., Heckley, W., Pailleux, J., Vasiljeric, D., Hamrud, M., Hollingsworth, A., Rabier, F., and Fisher, M.: The ECMWF implementation of three-dimensional variational assimilation (3D-Var). I: Formulation., Q. J. R. Meteorol. Soc., 124, 1783-1807, 1998.

Gustafsson, N., Berre, L., Hornqvist, S., Huang, X. Y., Lindskog, M., B.Navascue, Mogensen, K. S., and Thorsteinsson, S.: Three-dimensional variational data assimilation for a limited areamodel. Part I: General formulation and the background error constraint, Tellus, 53A, 425-446, 2001.

Di Michele, S., Tassa, A., Mugnai, A., Marzano, F. S., Bauer, P., and Poiares Baptista, J. P. V.: Bayesian Algorithm for Microwavebased Precipitation Retrieval: description and application to TMI measurements over ocean, IEEE Trans. Geosci. Rem. Sens., 43, 778-791, 2005.
Dudhia, J.: A nonhydrostatic version for the Penn-State-NCAR mesoscale model: Validation test and simulation of an Atlantic cyclone and cold front, Mon. Wea. Rev., 121, 1493-1513, 1993.

Grell, G. A., Dudhia, J., and Stauffer, D. R.: A description of fifth-generation Penn State/NCAR mesoscale model (MM5), Nat. Center Atmos. Res., Boulder, CA, NCAR Tech. Note NCAR/TN-398+STR, 1994.

Hollinger, J., Peirce, J., and Poe, G.: SSM/I Instrument Evaluation, IEEE Trans. Geosci. Remote Sens., 28, 781-790, 1990.

Kummerow, C., Hong, Y., Olson, W. S., Yang, S., Adler, F. R., McCollum, J., Ferraro, R., Petty, G., Shin, D. B., Wilbeit, T. T.: The evolution of Goddard Profiling algorithm (GPROF) for rainfall estimation from passive microwave sensors, J. Appl. Meeteorol., 40, 1801-1820, 2001.

Liebe, H.: An updated model for millimeter wave propagation in moist air, Radio Sci., 20, 1069-1089, 1985.

Marshall, J. and Palmer, W.: The distribution of raindrops with size, J. Meteorol., 5, 165-166, 1948.

Marzano, F. S., Fionda, E., and Ciotti, P.: Simulation of radiometric and attenuation measurements along earth-satellite links in the 10 to $50 \mathrm{GHz}$ band through horizontally-finite convective raincells, Radio Sci., 34, 841-858, 1999a.

Marzano, F. S., Mugnai, A., Panegrossi, G., Pierdicca, N., Smith, E. A., and Turk, J.: Bayesian estimation of precipitating cloud parameters from combined measurements of spaceborne microwave radiometer and radar, IEEE Trans. Geosci. Remote Sens., 37, 596-613, 1999b.

Marzano, F. S., Fionda, E., Ciotti, P., and Martellucci, A.: Groundbased multi-frequency microwave radiometry for rainfall remote sensing, Trans. Geosci. Rem. Sens., 40, 742-759, 2002.

Petty, G. W. and Katsaros, K. B.: The response of the Special Sensor Microwave/Imager to the marine environment. Part I: An analytic model for the atmospheric component of observed brightness temperatures, J. Atmos. Ocean. Tech., 9, 746-761, 1992.

Petty, G. W.: An intercomparison of oceanic precipitation frequencies from $10 \mathrm{SSM} / \mathrm{I}$ rain rate algorithms and shipboard presentweather reports, J. Geophys. Res., 102, 1757-1777, 1997.

Reisner, J., Rasmussen, R. J., and Bruintjes, R. T.: Explicit forecasting of supercooled liquid water in winter storms using the MM5 mesoscale model, Q. J. R. Meteorol. Soc., 124B, 10711107, 1998.

Smith, E. A., Bauer, P., Marzano, F. S., Kummerow, C. D., McKague, D., Mugnai, A., and Panegrossi, G.: Intercomparison of microwave radiative transfer models for precipitating clouds, Trans. Geosci. Remote Sens., 40, 197-203 2002.

Tao, K.-W. and Simpson, J.: Goddard Cumulus Ensemble Model. Part I: Model Description, TAO, 4, 35-72, 1993.

Tassa, A., Di Michele, S., Mugnai, A., Marzano, F. S., and Poires Baptista, J. P. V.: Cloud model-based Bayesian technique for precipitation profiles retrieval from Tropical Rainfall Measuring Mission Microwave Imager, Radio Sci., 38, 4, pp. 8074, 2003.

Ulaby, F. T., Moore, R. K., and Fung, A. K.: Microwave remote sensing active and passive, in: Microwave Remote Sensing Fundamentals and Radiometry, edited by: Reading, M. A., Addison Wensley, 1, 1981. 